\title{
Pulsation of the $\delta$ Scuti multiple system DG Leo
}

\author{
P. Lampens ${ }^{1}$, Y. Frémat ${ }^{1}$, R. Garrido ${ }^{2}$, J.H. Peña ${ }^{3}$, L. Parrao ${ }^{3}$, \\ P. Van Cauteren ${ }^{4}$, J. Cuypers ${ }^{1}$, P. De Cat ${ }^{1}$, H. Hensberge ${ }^{1}$, \\ T. Arentoft ${ }^{5}$, P. Mathias ${ }^{6}$ and M. Hobart ${ }^{7}$ \\ ${ }^{1}$ Royal Observatory of Belgium, Ringlaan 3, B-1180 Brussel, Belgium \\ ${ }^{2}$ Instituto de Astrofísica de Andalucía Camino Bajo de Huetor 24, 18008 Granada, España \\ ${ }^{3}$ Instituto de Astronomía, UNAM Apartado Postal 70-264 México 04510, D.F. \\ ${ }^{4}$ Beersel Hills Observatory, Laarheidestraat 166, B-1650 Beersel, Belgium \\ ${ }^{5}$ Department of Physics and Astronomy, Aarhus University, DK-8000 Aarhus C., Denmark \\ ${ }^{6}$ Observatoire de la Côte d'Azur, UMR 6528, F-06304 Nice Cedex 4, France \\ ${ }^{7}$ Facultad de Fisica, Universidad Veracruzana, A. Postal 270, Xalapa, Veracruz, Mexico
}

\begin{abstract}
DG Leo is a spectroscopic triple system showing $\delta$ Scuti type photometric and spectroscopic variations. The three components have nearly equal masses but different chemical compositions in the outer layers. All three are potential pulsators. Frequency analyses of the photometric data were carried out using various methods. These global results together with those of the spectroscopic analysis permit us to discuss of the behaviour of each component with respect to pulsation.
\end{abstract}

Keywords. Binaries: spectroscopic, stars: variable: $\delta$ Sct, stars: individual: (DG Leo), stars: oscillations (including pulsations)

\section{Introduction}

A bonus to the study of pulsating stars in binary and multiple systems is that one can exploit the dynamical information to obtain independent information on the physical properties to gather more constraints on the pulsational models. Multi-year photometry with a good phase coverage of the beat periods of the oscillations permit, on one hand, an accurate frequency analysis. A careful spectroscopic analysis, on the other hand, may provide information on the mass and luminosity ratios, the effective temperature, the metallicity and the superficial gravity of each component provided the spectrum is composite. By studying and comparing the pulsational content of the components which originate from the same protostellar environment, one may realistically hope to obtain clues to better understand the pulsation physics since a difference in pulsational behaviour between each can only be attributed to a limited number of (differing) stellar parameters or physical processes.

In a review of $\delta$ Scuti stars in known double or multiple stars, Lampens \& Boffin (2000) discussed several potentially interesting objects, one of which was DG Leo which appears to be a triple system with all three components potential pulsators. Fundamental parameters of the components are discussed in (Frémat et al. 2005) while we discuss their variations by confronting photometric with spectroscopic results. 


\section{Photometry}

Photoelectric photometry campaigns were carried out during 2002, 2003, and 2004. Most of the data was collected during a few weeks at two good sites located at different longitudes and equipped with identical instrumentation and standard Strömgren filters: namely the Observatories of San Pedro Martír (OAN), México, and Sierra Granada (OSN), Spain. Additional CCD differential measurements were obtained at Beersel Hills Observatory (BHO), Belgium, cover a period of several months in 2003. The analysis of these data (based on a total of 2514 measurements) shows that most of the photometric variability can be modelled by a composite sine wave with at least four frequencies, as illustrated and detailed in Fig. 1 (Lampens et al. 2005).

In DG Leo's photometry the highest amplitude variation is related to the tidal deformation (ellipsoidal variation) of the close binary components (Fig. 1a). Its periodicity corresponds to one-half of the 4.15 day orbital period of the close binary. Three other frequencies having close periods of about 2 hours were found to be caused by pulsation (Fig. 1b) of at least one of the components of the triple system.

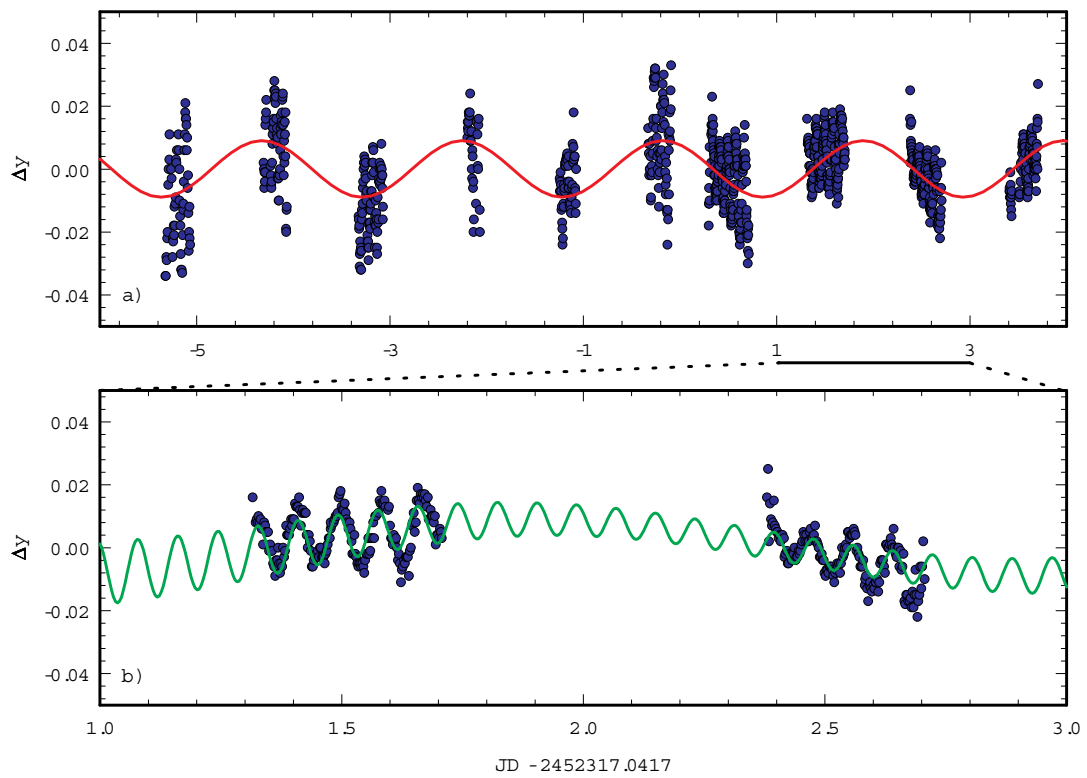

Figure 1. a) Predictions (assuming a sinusoidal variation) for the ellipsoidal variations (red curve) are compared to the observations (blue dots). b) We compare the predictions from the combined solution (ellipsoidal variation+pulsation) of the frequency analysis (green line) to some light curves in the y colour band obtained during 2003 (blue dots).

\section{Spectroscopy}

The acquisition of the spectroscopic data is described in Frémat et al. (2005). The spectral disentangling that was carried out using the KOREL computer code (Hadrava 1995) allowed us to determine the radial velocities of the components even at phases where their contributions cannot be spectroscopically resolved by traditional techniques. Significant short-term ( $\sim$ few hours) variations are detected for component B only (see Fig. 2b). 


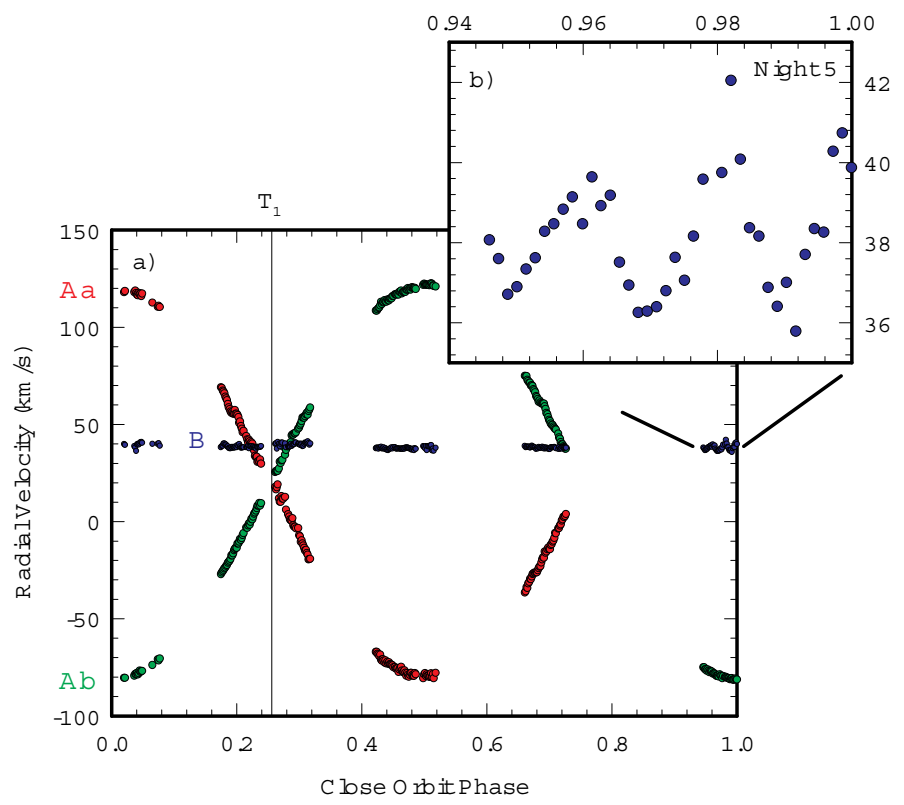

Figure 2. Radial velocities are reported against the orbital phase of the spectroscopic binary. $\mathrm{T}_{1}$ stands for the epoch at which both $\mathrm{A}$ components have the same radial velocity.

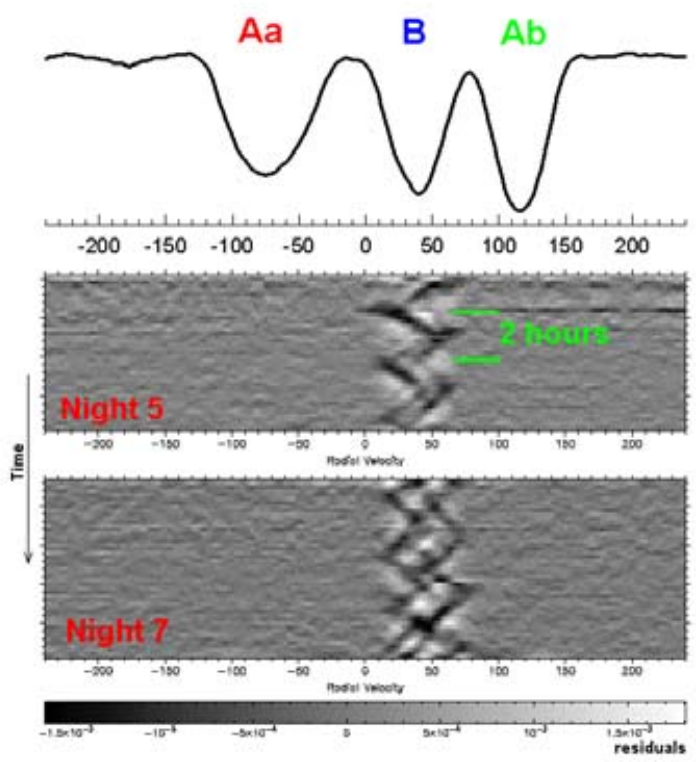

Figure 3. Residuals of the instantaneous cross correlation functions reported against time.

After each exposure, the INTERTACOS pipeline (Baranne et al. 1996) was used to reduce the data and further provided us with a cross-correlation function (CCF) computed using a F0 V line-template. These CCFs can be seen as the mean profile of about 2000 lines taken over the whole spectral domain covered by the ELODIE spectrograph. Strong 
variations of the cross-correlation peak corresponding to DG Leo B were detected. The residuals of the instantaneous cross-correlation functions (upper panel in Fig. 3, where each peak represents the contribution of one component) show that the spectroscopic variations are restricted to component B as they are only centered on its correlation peak. Their period is of the order of 2 hours. Line profile variations (LPVs) are observed every night but their pattern changes from night to night. During Night 5 (lower panel of Fig. 3) LPVs are mainly affecting the apparent radial velocity (see also Fig. 2) while in Night 7 LPVs are mostly appearing as bumps moving through the line. These differences are clear evidence of a multiperiodic pulsation.

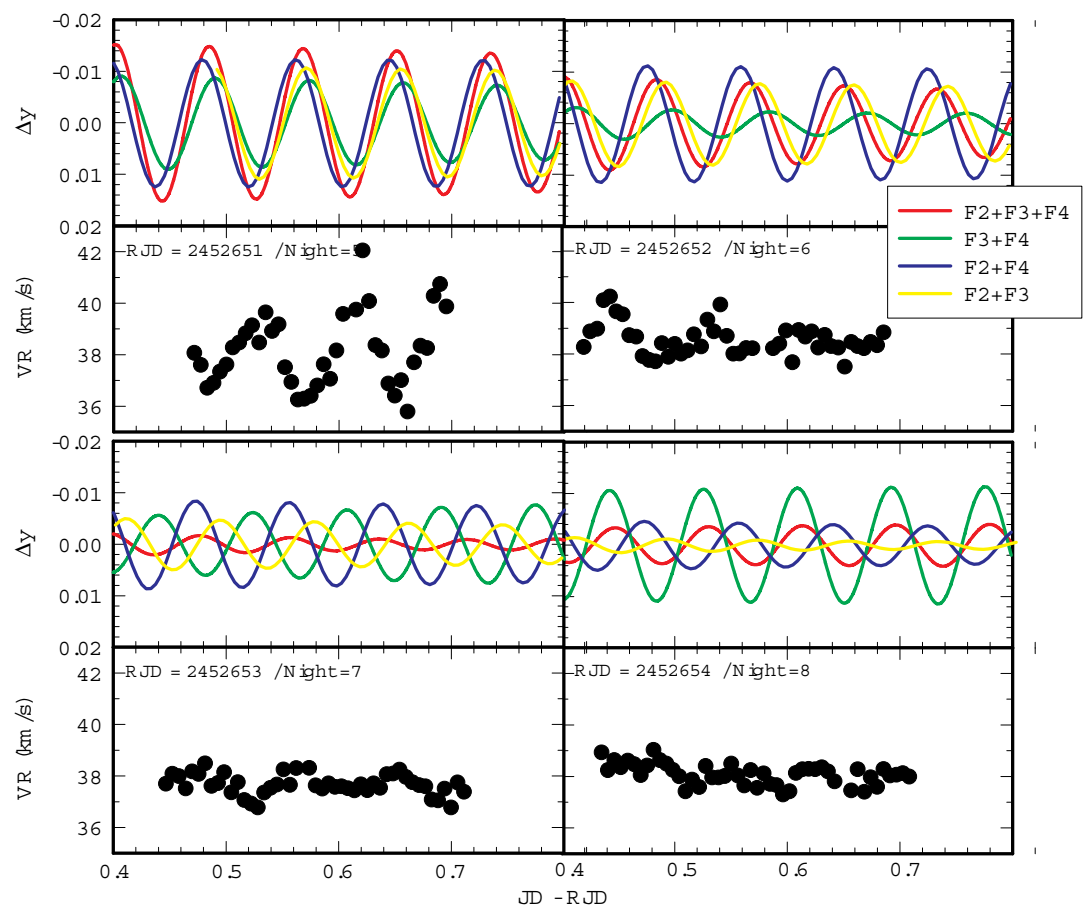

Figure 4. The light curves are plotted as predicted from the 3-frequency solution (in red) as well as those predicted from the various 2-frequency combinations (in resp. green, blue, yellow) together with the radial velocity variations of component B as derived from the spectral disentangling technique.

\section{Spectroscopy \& photometry}

The frequency solution obtained from the photometric data (without considering the ellipsoidal variation, i.e., F1) (see Fig. 1) was used to predict the light curves during those nights for which high-resolution spectra are available. In Fig. 4 we plotted the light curves as predicted from the 3-frequency solution (in red) as well as those predicted from the various 2-frequency combinations (in resp. green, blue, yellow) together with the radial velocity variations of component $\mathrm{B}$ as derived from the spectral disentangling technique. At a predicted epoch of maximum beat amplitude, the observed amplitude in radial velocity is the highest (Night 5) while it decreases rapidly following a similar decrease in beat amplitude (Night 6) and becomes insignificant the next two nights (Nights 7 and 8), when the beat amplitude is minimum. We further note that the radial velocity variations 
during Night 5 are in anti-phase with the predicted light curve, a behaviour typical of $\delta$ Scuti variable in the presence of low degree modes. A preliminary conclusion is that at least two photometrically detected frequencies may be associated with component B. The next logical step will be to perform a frequency analysis of the spectroscopic data and directly compare its results to those derived from the photometry.

\section{Acknowledgements}

This work is based on observations made at the Haute-Provence Observatory (France). YF and PL acknowledge funding from the Belgian Federal Science Policy (Research project MO/33/007) and from the FNRS (Travel grant to Dubrovnik). HH acknowledges support from the IAP P5/36 project of the Belgian Federal Science Policy.

\section{References}

Baranne, A., Queloz, D., Mayor, M., et al. 1996, A\&AS, 119, 373

Frémat, Y., Lampens, P., \& Hensberge, H. 2005, These Proceedings, FP12

Hadrava, P. 1995, A\&AS, 114, 393

Lampens, P. \& Boffin, H. M. J. 2000, in ASP Conf. Ser. 210: Delta Scuti and Related Stars, 309

Lampens, P., Garrido, R., Parrao, L., et al. 2005, IAU Colloquium 191 held in Merida (Mexico), Feb. 2003, eds. C. Allen \& C. Scarfe, Rev. Mex. A.A., in press

Zahn, J. P. 1992, in Binaries as Tracers of Stellar Formation, eds: A. Duquennoy, M. Mayor, Cambridge University Press, 253 